\title{
Progressive bradykinesia and hypokinesia of ocular pursuit in Parkinson's disease
}

\author{
G U Lekwuwa, G R Barnes, C J S Collins, P Limousin
}

\begin{abstract}
Objectives-Patients with Parkinson's disease characteristically have difficulty in sustaining repetitive motor actions. The purpose of this study was to establish if parkinsonian difficulty with sustaining repetitive limb movements also applies to smooth ocular pursuit and to identify any pursuit abnormalities characteristic of Parkinson's disease.
\end{abstract}

Methods-Ocular pursuit in seven patients with moderate to severe bradykinesia predominant Parkinson's disease was compared with seven age matched controls. Predictive and non-predictive pursuit of constant velocity target ramps were examined. Subjects pursued intermittently illuminated $40 \%$ ramps sweeping to the left or right with an exposure duration of $480 \mathrm{~ms}$ and average interval of $1.728 \mathrm{~s}$ between presentations. To examine for any temporal changes in peak eye velocity, eye displacement or anticipatory smooth pursuit the $124 \mathrm{~s}$ duration of each record was divided into four epochs (E1, E2, E3, E4), each lasting 31 s and containing 18 ramp stimuli. Three test conditions were examined in each subject: predictive (PRD1), non-predictive (NPD), and predictive (PRD2) in that order.

Results-Both patients and controls initiated appropriate anticipatory pursuit before target onset in the PRD1 and PRD2 conditions that enhanced the response compared with the NPD condition. The distinctive findings in patients with Parkinson's disease were a reduction in response magnitude compared with controls and a progressive decline of response with stimulus repetition. The deficits were explained on the basis of easy fatiguability in Parkinson's disease.

Conclusions-Ocular pursuit shows distinct anticipatory movements in Parkinson's disease but peak velocity and displacement are reduced and progressively decline with repetition as found with limb movements.

(f Neurol Neurosurg Psychiatry 1999;66:746-753)

Keywords: ocular pursuit; Parkinson's disease; bradykinesia; hypokinesia; prediction

Patients with Parkinson's disease characteristically have difficulty initiating movements (akinesia). When movements are initiated, they are of low velocity (bradykinesia), and reduced amplitude (hypokinesia). ${ }^{12}$ In addition, patients with Parkinson's disease are unable to sustain repetitive motor action. When they attempt to open or close the hand rapidly or tap the foot on the ground, the movement rapidly decreases in amplitude and slows in speed until it ceases. ${ }^{2}$ This disability is easily appreciated in the progressive micrographia of the handwriting of parkinsonian patients.

Research in the past 30 years has established that Parkinson's disease impairs control of eye movements. ${ }^{3-16}$ Voluntary saccades, such as self paced, predictive, and remembered saccades are hypometric, multistep, of reduced velocity, and of increased duration. Visually guided saccades are normal. An oculomotor pathway is known to form one of the basal ganglia loops. ${ }^{17}$ The role of the basal ganglia in the control of saccadic eye movements has been extensively reviewed by Hikosaka. ${ }^{18}$ The role of the basal ganglia seems to be a selective gating of reflexive or voluntary saccades generated by the superior colliculus. No study has as yet defined any ocular smooth pursuit pathway through the basal ganglia. Advanced Parkinson's disease is known to be associated with reduced ocular smooth pursuit gain. ${ }^{11}$ This has been explained on the grounds that advanced Parkinson's disease affects other structures outside the basal ganglia. ${ }^{3}$ Reduced ocular pursuit gain is a non-specific finding in many cerebral, brainstem, and cerebellar disorders. It is not characteristic of Parkinson's disease or other neurodegenerative disorders. In this paper we show that the characteristic temporal decay in the velocity and amplitude associated with limb and other skeletal movements in Parkinson's disease also applies to smooth pursuit eye movements. We also use the pursuit of intermittently presented constant velocity target motion stimuli (ramps) ${ }^{19} 20$ to show that patients with severe Parkinson's disease are still able to generate appropriate anticipatory smooth eye movements. The anticipatory and visually guided pursuit eye movements of bradykinetic parkinsonian patients are compared with those of control subjects.

\section{Methods}

SUBJECTS

We studied ocular pursuit in seven patients with moderate to severe bradykinesia predominant Parkinson's disease (mean age 55.9 years, range 48-66 years) and seven age matched controls (mean age 53.4 years, range 40-80 years). All patients had idiopathic Parkinson's disease without dementia, and were receiving treatment at the National Hospital for Neurology and Neurosurgery, Queen Square, London (table 1). Motor disability was evaluated using the motor subscale of the unified Parkinson's 
Table 1 Clinical characteristics of patients with Parkinson's disease

\begin{tabular}{llllll}
\hline Patient & Age (y) & Sex & $\begin{array}{l}\text { Bradykinesia } \\
\text { rating } \\
\text { (UPDRS) }\end{array}$ & $\begin{array}{l}\text { Illness } \\
\text { duration }(y)\end{array}$ & Medication \\
\hline 1 & 66 & $\mathrm{M}$ & 4 & 13 & Madopar, apomorphine, pergolide \\
2 & 53 & $\mathrm{M}$ & 4 & 6 & Sinemet, pergolide, apomorphine, \\
3 & 60 & $\mathrm{~F}$ & 4 & 17 & Sinemet, apomorphine \\
4 & 48 & $\mathrm{M}$ & 4 & 9 & Sinemet, pergolide \\
5 & 48 & $\mathrm{M}$ & 3 & 15 & Madopar, amitriptyline \\
6 & 62 & $\mathrm{~F}$ & 3 & 36 & Madopar, pergolide, fluoxetine \\
7 & 54 & $\mathrm{M}$ & 3 & 12 & Selegeline, sinemet, pergolide \\
\hline
\end{tabular}

disease rating scale (UPDRS). ${ }^{21}$ All patients scored 3-4 (maximum score) on the bradykinesia rating items of the UPDRS. Duration of disease ranged between 6 and 36 years with a mean duration of 15.4 years. Patients were studied 12 hours after withdrawal of medication. Informed consent was obtained from each subject and experiments were approved by the local ethics committee.

RECORDING METHODS

The subjects were seated in the centre of a darkened room in front of a semicircular screen of radius $1.5 \mathrm{~m}$. Horizontal eye movements were recorded using an infrared limbus reflection technique (Iris 6500 system, Skalar Medical) with a resolution of 5-10 minutes of arc,
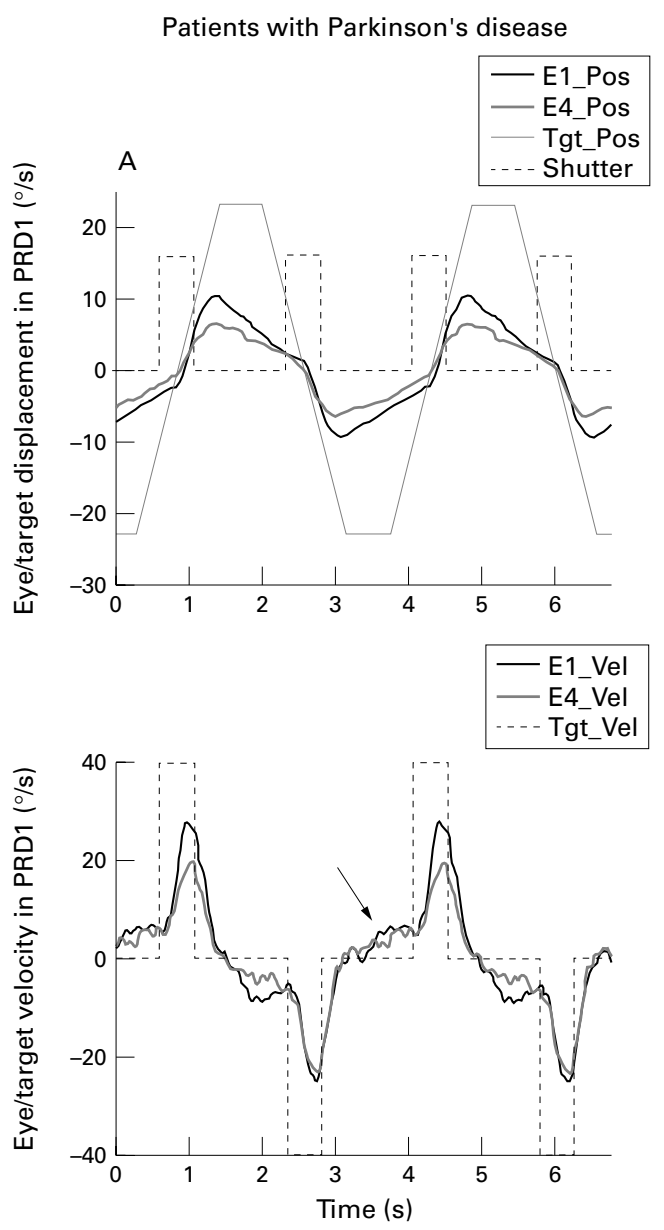

Figure 1 Profiles of mean eye displacement (upper traces) and eye velocity (lower traces) in the PRD1 condition averaged across the seven patients with Parkinson's disease $(A)$ and seven control subjects (B). Each cycle of average has been plotted twice to facilitate viewing. Right going movements are positive. Shutter signal indicates time of presentation of target. Target velocity pulses indicate ramp velocity and duration of target exposure (480 ms). Responses to first and fourth epochs only are plotted for eye displacement (E1_pos, E4_pos) and velocity (E1_vel, E4_vel). Both groups initiated anticipatory pursuit before onset of target motion (arrows).

and a linear range of at least $\pm 20^{\circ}$. Recorded data were passed through a low pass analogue filter with a cut off frequency of $80 \mathrm{~Hz}$. The eye movement recorders were mounted on a helmet assembly which was attached firmly to the subject's head. The head was fixed by padded clamps that fitted snugly on both sides of the head. Subjects were instructed to track targets using only eye movements.

\section{STIMULUS}

The stimulus consisted of a circle of diameter 50 minutes of arc with superimposed cross hairs. It was made to move across the screen in the horizontal plane by a mirror galvanometer. The motion of the target, which varied from one experiment to the other, was controlled by a computer generated signal. Target appearance was controlled by an electromechanical shutter. Eye movements were calibrated before each record using a simple sinusoidal target waveform at $0.2 \mathrm{~Hz}$. Data were sampled at 125 $\mathrm{Hz}$.

EXPERIMENTAL PROCEDURE: PREDICTIVE/NONPREDICTIVE RAMP PURSUIT

In the predictive paradigm, subjects pursued an intermittently illuminated $40 \%$ s constant velocity target (ramp) sweeping alternately to
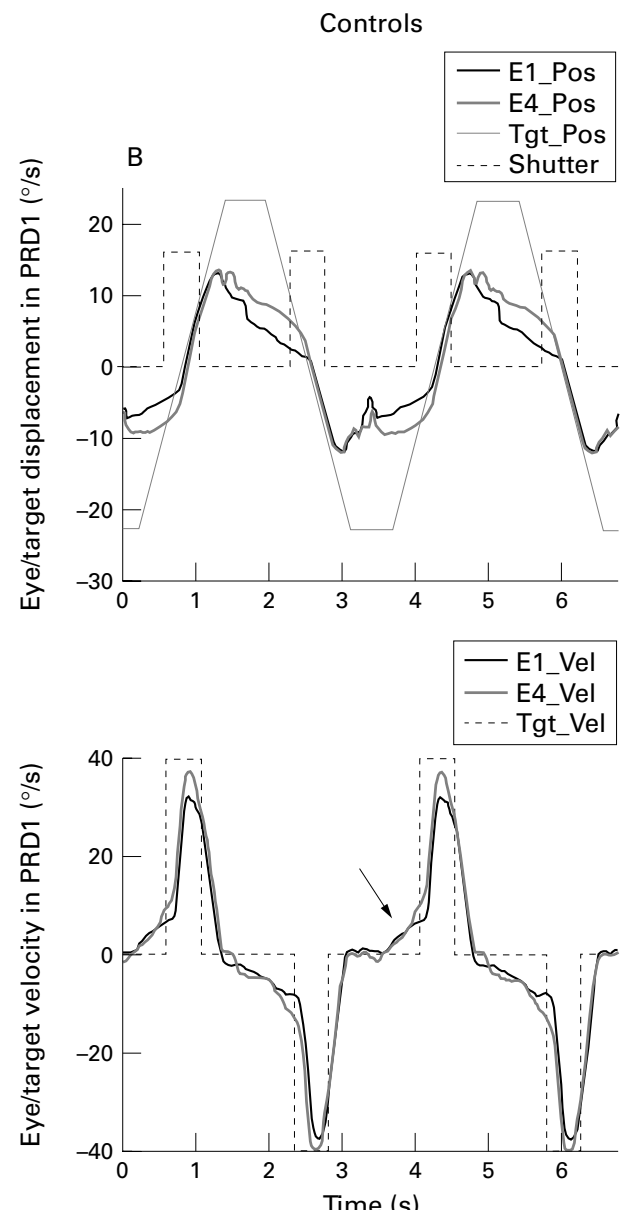
Patients with Parkinson's disease
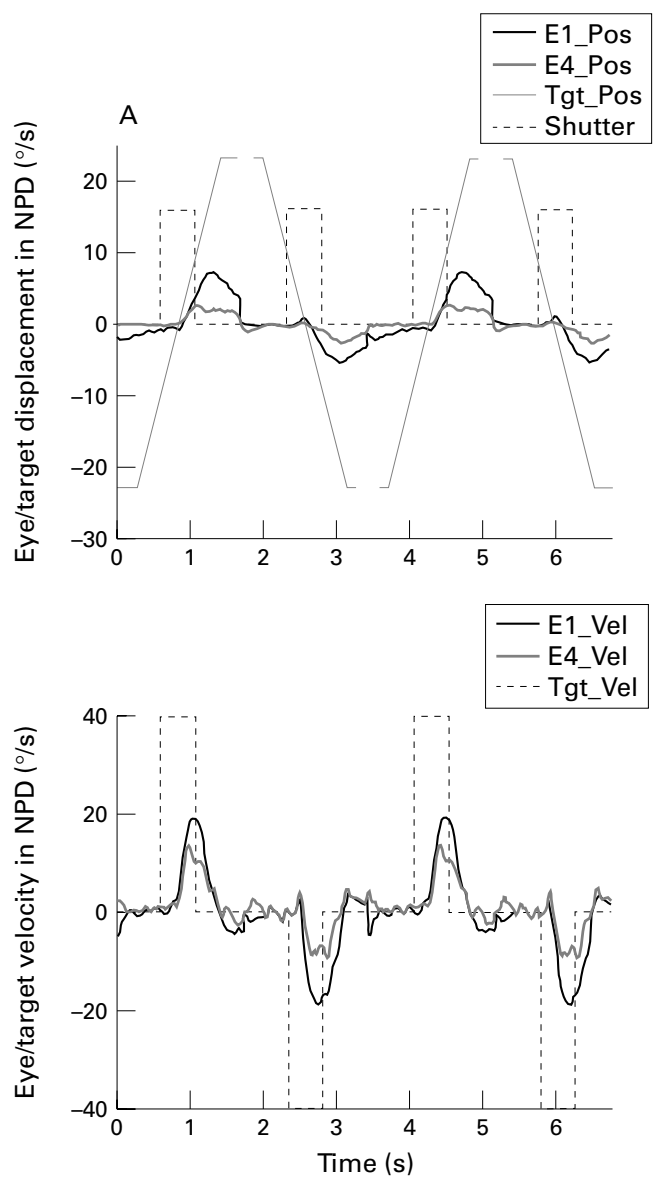

Controls
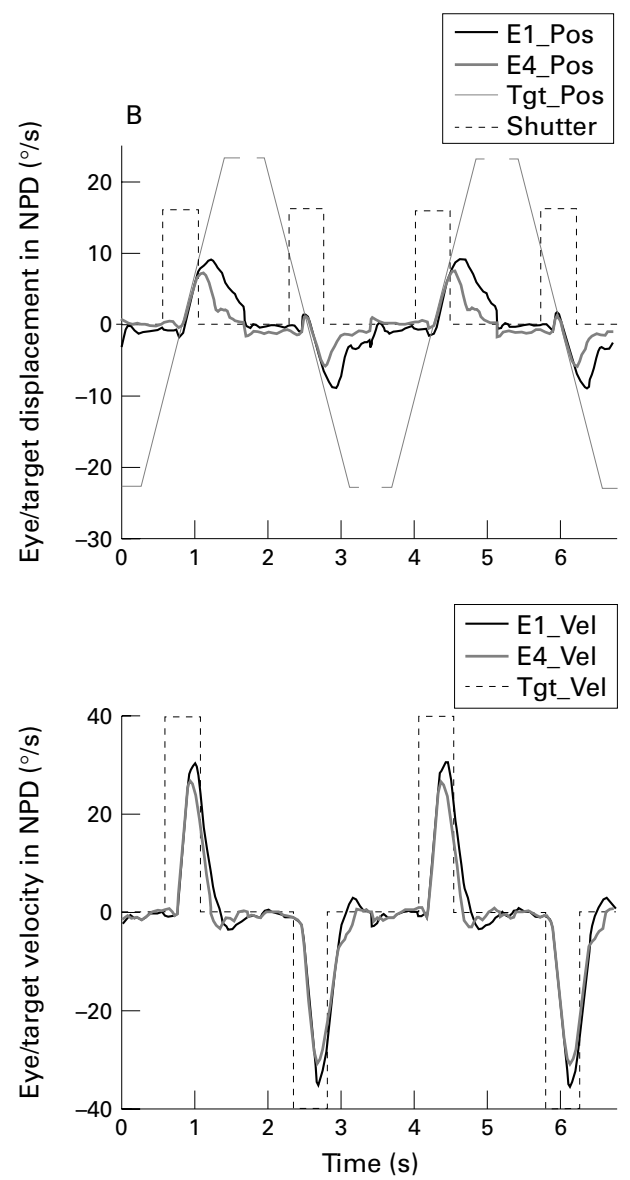

Figure 2 Profiles of mean eye displacement (upper traces) and eye velocity (lower traces) in the NPD condition averaged across the seven patients with Parkinson's disease $(A)$ and seven control subjects $(B)$. Averages of right and left going responses derived from individual ramp stimuli of randomised direction within each epoch. Break in target displacement indicates that right and left going stimuli were not consecutive. Other details as in fig 1.

the left and right with a period of $3.456 \mathrm{~s}$. Target exposure duration was $480 \mathrm{~ms}$ and its presentation was timed so that the midpoint of the presentation period occurred as the target passed through the central position. The peak to peak target displacement was $19.2^{\circ}$. The extinction interval between presentations was $1.248 \mathrm{~s}$. Each record lasted about 2 minutes $(124.4 \mathrm{~s})$ and consisted of 36 pairs of alternate velocity ramps. In the non-predictive paradigm, the target parameters were the same as in the predictive parameters, except that the target direction and the interpresentation intervals were randomised (range 1.296 $\mathrm{s}-2.160 \mathrm{~s}$ ); the average interpulse interval of $1.728 \mathrm{~s}$ remained the same in both paradigms. To eliminate the effect of fatigue as a confounding variable in the causation of any differences found between the predictive and non-predictive paradigms, three test conditions were examined in each subject: predictive (PRD1), non-predictive (NPD), and predictive (PRD2) in that order, with a time interval of 2-3 minutes between conditions.

ANALYSIS

All records were analysed off line by computer using an interactive computer graphics procedure described by Barnes ${ }^{22}$ to remove the saccadic components and thus obtain the smooth eye velocity trajectories. In the description of the results, the term eye velocity refers only to the smooth component of eye movement, whereas eye displacement refers to the original eye movement signal before the removal of saccades. Because of the problem of defining a peak level of displacement in the presence of saccadic activity, the magnitude of eye displacement was defined in terms of its root mean square (RMS) amplitude. Anticipatory eye movements refer to smooth eye movements initiated before the onset of target motion (figs 1,2). In all measures indicated in the figures, movements to the right are positive and movements to the left are negative.

To show more clearly the temporal changes in the eye movement response, we divided the $124 \mathrm{~s}$ duration of each pursuit record into four consecutive epochs (E1, E2, E3, E4), each epoch lasting $31 \mathrm{~s}$ and containing 18 velocity ramps (nine in each direction). Within each of these epochs, five principal measures of response were made for each ramp presentation: (a) peak eye velocity; (b) RMS eye displacement; (c) the magnitude of anticipatory velocity (V100), which was assessed by measuring eye velocity $100 \mathrm{~ms}$ after target onset (at the time that visual feedback would be expected to become effective); (d) eye velocity $200 \mathrm{~ms}$ after target onset, and (e) the latency 
Patients with Parkinson's disease
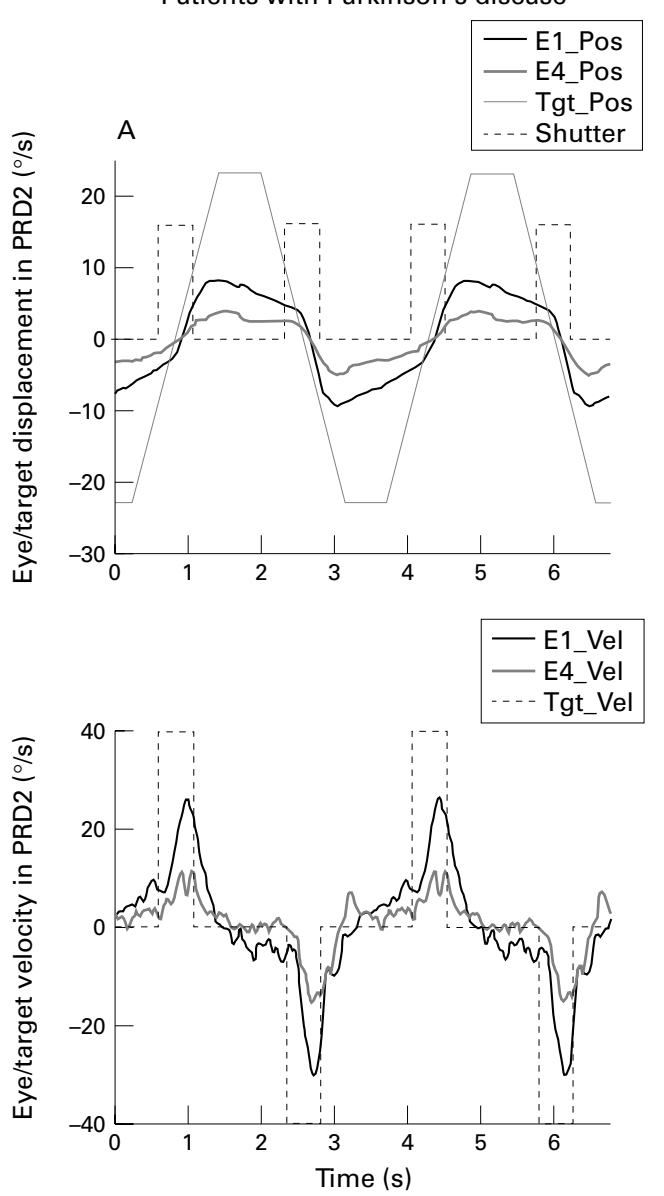
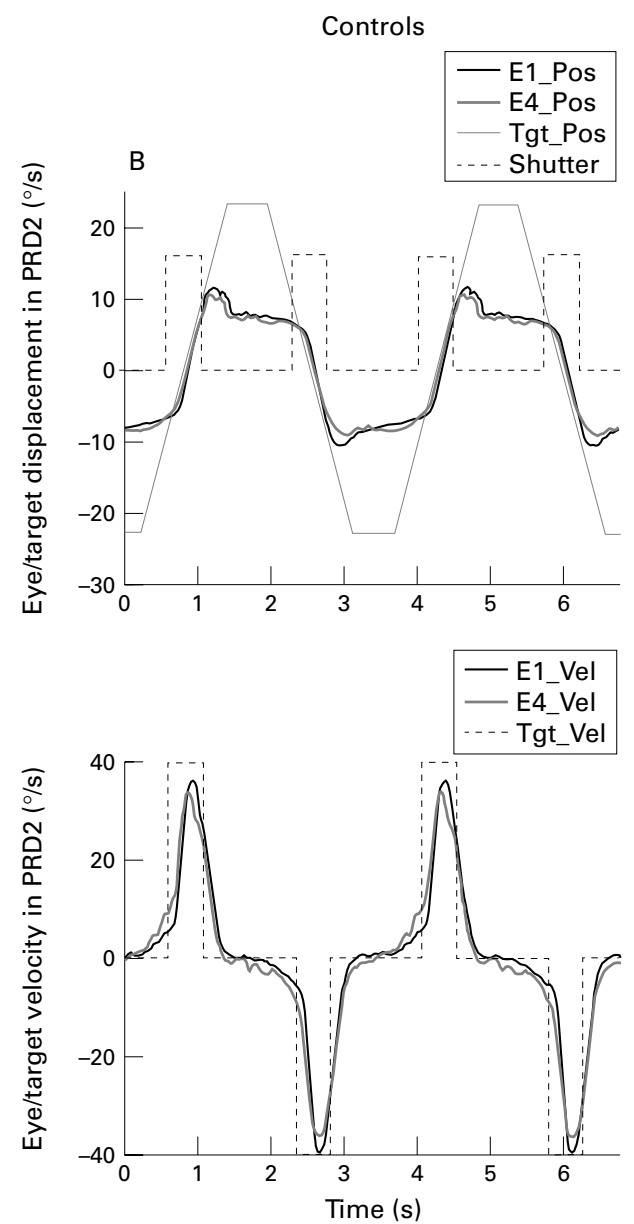

Figure 3 Profiles of mean eye displacement (upper traces) and eye velocity (lower traces) in the PRD2 condition averaged across the seven patients with Parkinson's disease $(A)$ and seven control subjects $(B)$. Other details are as in fig 1.

between onset of target appearance and onset of smooth pursuit (measured by using techniques described previously ${ }^{20}$ ). In addition, we assessed RMS velocity within each cycle as this formed a more robust measure of average velocity, whereas peak velocity was sometimes ill defined in the patients.

Statistical data analysis was carried out using SPSS software. Before performing analyses of variance (ANOVAs), the data were tested for normality (Shapiro-Wilk test), homogeneity of variance between patients and control groups (Levene test), and homogeneity of covariance matrices between groups (Box test). The results showed that the data were normal and variances (and covariance matrices) did not differ in the two groups. Movements to left and right were averaged, as preliminary ANOVAs on all variables showed no significant effect of direction.

\section{Results}

GENERAL RESPONSE FEATURES

The predictive paradigm (PRD1 and PRD2 conditions) used here elicited a very characteristic pattern of eye movement that has been described previously. ${ }^{19}$ During the first one or two presentations of the target, the eye movements were initiated some 100-150 ms after target onset in control subjects, but with further repetition of the stimulus, anticipatory eye movements started to build up before target onset. A steady state was generally reached after three to four presentations and this can be represented by averaging the subsequent responses to obtain displacement and velocity profiles of the type shown in figs 1 and 2 . In general, the number of saccades was very small in the steady state phase from the time at which the anticipatory smooth movement was initiated until some $200 \mathrm{~ms}$ after target offset. However, it should be emphasised that in obtaining the average displacement profiles, any saccades (usually very small) have not been removed. Nevertheless, it is not difficult to see that in the controls the eye followed the target rather closely from shortly after target onset until target disappearance. By contrast, the patients did not make such large amplitude movements even in the first epoch and this had diminished even more by the fourth epoch.

All the patients with Parkinson's disease were able to initiate appropriately directed anticipatory pursuit movements before the onset of target motion in the predictive pursuit paradigm in the same way as the controls (figs 1 and 2), although qualitatively the pattern was slightly different. Essentially, patients with Parkinson's disease seemed to have more difficulty in sustaining the continued increase in eye velocity in this anticipatory phase, so that there was a more abrupt transition between the 
Table 2 Results of two way ANOVA for the specified variables (peak velocity, RMS displacement, V100, V200, and onset latency) averaged across all epochs.

\begin{tabular}{|c|c|c|c|c|c|}
\hline $\begin{array}{l}\text { Two way ANOVA: } \\
\text { factors: group and } \\
\text { condition }\end{array}$ & Peak velocity & $R M S$ displ & V100 & $V 200$ & Onset latency \\
\hline \multicolumn{6}{|l|}{ Group comparison: } \\
\hline PD $v$ Control & $F=12.786$ & $F=10.461$ & $F=6.968$ & $F=18.529$ & $F=1.373$ \\
\hline$(\mathrm{d} F=1,12)$ & $\mathrm{p}=0.004^{\star}$ & $\mathrm{p}=0.007^{\star}$ & $\mathrm{p}=0.022^{\star}$ & $\mathrm{p}=0.001^{\star}$ & $\mathrm{p}=0.264$ \\
\hline \multicolumn{6}{|l|}{ Contrasts: } \\
\hline PRD1 $v \quad$ PRD2 & $F=0.507$ & $F=3.183$ & $F=0.505$ & $F=0.372$ & $F=11.707$ \\
\hline$(\mathrm{d} F=1,12)$ & $\mathrm{p}=0.49$ & $\mathrm{p}=0.100$ & $\mathrm{p}=0.491$ & $\mathrm{p}=0.859$ & $\mathrm{p}=0.005^{\star}$ \\
\hline Condition $\times$ group & $F=0.494$ & $F=0.165$ & $F=0.03$ & $F=0.531$ & $F=1.098$ \\
\hline$(\mathrm{d} F=1,12)$ & $\mathrm{p}=0.495$ & $\mathrm{p}=0.691$ & $\mathrm{p}=0.865$ & $\mathrm{p}=0.416$ & $\mathrm{p}=0.315$ \\
\hline PRD $1 v$ NPD & $F=17.895$ & $F=47.888$ & $F=33.251$ & $F=57.093$ & $F=111.794$ \\
\hline$(\mathrm{d} F=1,12)$ & $\mathrm{p}=0.001^{\star}$ & $\mathrm{p}=1 \cdot 6 \cdot 10^{-5 \star}$ & $\mathrm{p}=0.0001^{\star}$ & $\mathrm{p}=1.10^{-5 \star}$ & $\mathrm{p}=2.10^{-7} \star$ \\
\hline Condition $\times$ group & $F=0.959$ & $F=2.655$ & $F=3.223$ & $F=5.082$ & $F=1.122$ \\
\hline$(\mathrm{d} F=1,12)$ & $\mathrm{p}=0.347$ & $\mathrm{p}=0.129$ & $\mathrm{p}=0.098$ & $\mathrm{p}=0.044^{\star}$ & $\mathrm{p}=0.310$ \\
\hline
\end{tabular}

The factors were group (patients with Parkinson's disease and controls) and condition (PRD1, $\mathrm{NPD}$, and PRD2). ${ }^{\star} \mathrm{p}<0.05$.
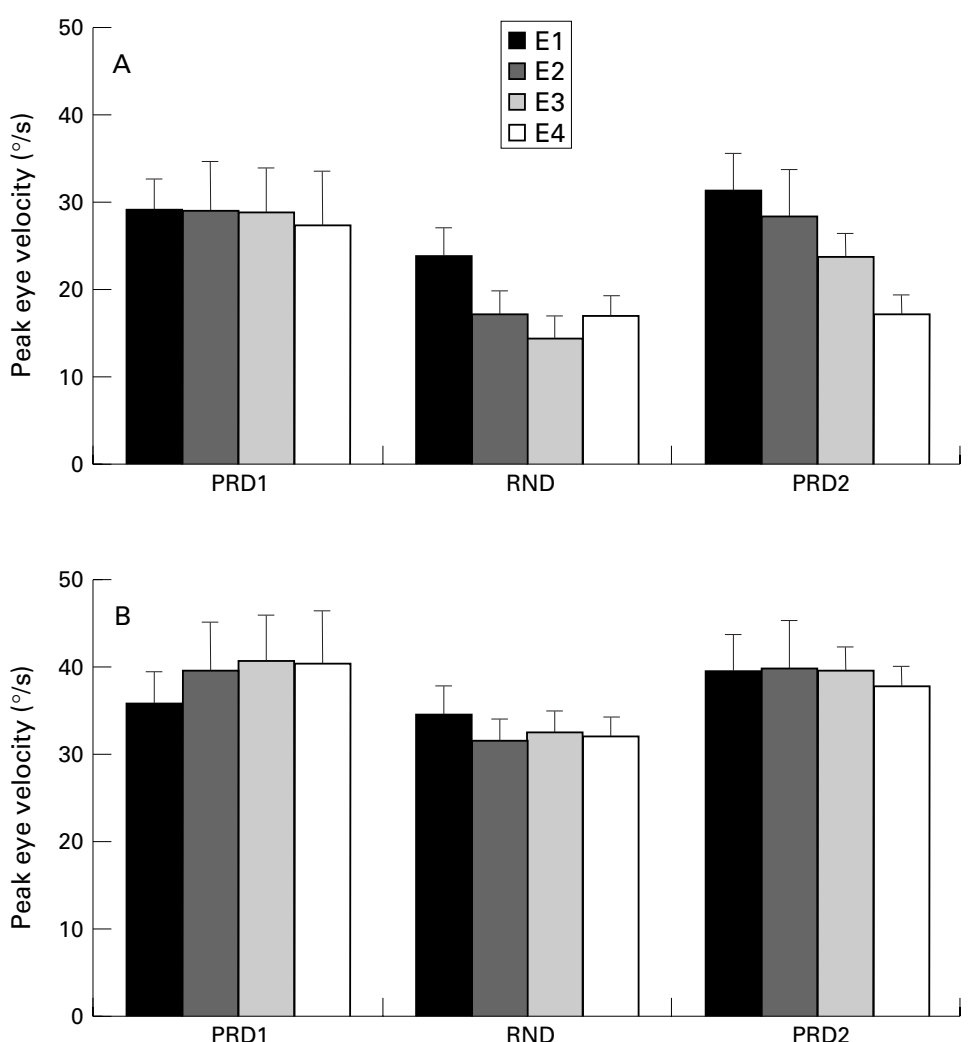

Figure 4 Peak eye velocity in the four epochs (E1-E4) of each stimulus condition (PRD1, NPD, PRD2) for controls and patients with Parkinson's disease. Each value is the mean (SEM). across nine responses in two directions for seven subjects

anticipatory movement and the subsequent part of the movement that was probably more dependent on visual feedback mechanisms (figs 1 and 2).

In the non-predictive paradigm there was an absence of any substantial anticipatory pursuit movements in both the controls and patients (fig 3), although occasional anticipatory guesses were evident. On average, in this paradigm, eye movements were not initiated until well after target onset and eye movement did not match the target as closely as in the PRD paradigm.

In the controls there was no obvious deterioration or fluctuation of the eye movement response throughout either the PRD1 or PRD2 conditions and velocity levels in PRD1 and PRD2 were comparable. However, in the patients there was an apparent decline in the magnitude of the response throughout the period of each stimulus condition, as shown by comparison of the responses in the first and last epochs (E1 and E4; fig 1). This effect was particularly evident in the PRD2 condition. This decline did not occur as a continuous process; there was a waxing and waning of the response that was more evident in some patients than in others. The clearest way to demonstrate this was to split the whole of each run into four equal, consecutive epochs (as described in the methods section) and compare the averaged measures of eye movement across these epochs.

To compare responses of patients with those of controls we carried out two types of analysis, looking firstly at differences between groups and test conditions and then at changes across epochs. In all cases, results for RMS velocity showed the same trend as for peak velocity, so these results will not be quoted.

\section{DIFFERENCES BETWEEN GROUPS AND TEST CONDITIONS}

To assess differences in absolute levels of all measured variables, two way ANOVAs were carried out in which the factors were group (control or Parkinson's disease) and test condition (PRD1, NPD, PRD2: averaged across epochs). Differences between test conditions were assessed by using a priori simple contrasts to compare PRD1 with NPD, and PRD1 with PRD2.

Values of peak velocity (fig 4), and RMS displacement were similarly affected by the two factors. Detailed results from this analysis are given in table 2. Overall, the values for patients with Parkinson's disease were significantly less than for controls. There was also a significant difference between PRD1 and NPD (PRD1>NPD), but no difference between PRD1 and PRD2. There was no interaction between group and paradigm showing that this pattern of results was the same in the control and Parkinson's disease groups.

Although patients with Parkinson's disease were able to make anticipatory smooth movements (fig 1) the velocity (V100) was significantly less than in the controls (fig 5; table 2). But V100 was still significantly greater in the PRD1 condition than in the NPD condition in the same way as for controls (table 2). Moreover, comparison of the velocity at $200 \mathrm{~ms}$ (V200) for the PRD1 and NPD conditions confirmed that the predictive response was still significantly greater than that in the randomised mode at this time (table 2). Note that although there was a significant interaction between paradigm and group for V200, subsequent analysis with one way ANOVA on the patients with Parkinson's disease alone confirmed that V200 was significantly greater for PRD1 than NPD $(F=15.4, \mathrm{df}=1,6 ; \mathrm{p}=0.008)$. This result indicates that although V100 was often quite low, it was, nevertheless, contributing to the predictive process by generating higher velocity earlier in the response that was then sustained until at least $200 \mathrm{~ms}$. In fact, in the PRD conditions, V100 formed a similar proportion of peak eye velocity in patients with Parkinson's disease $(27 \%)$ as it did in controls $(32 \%)$. 

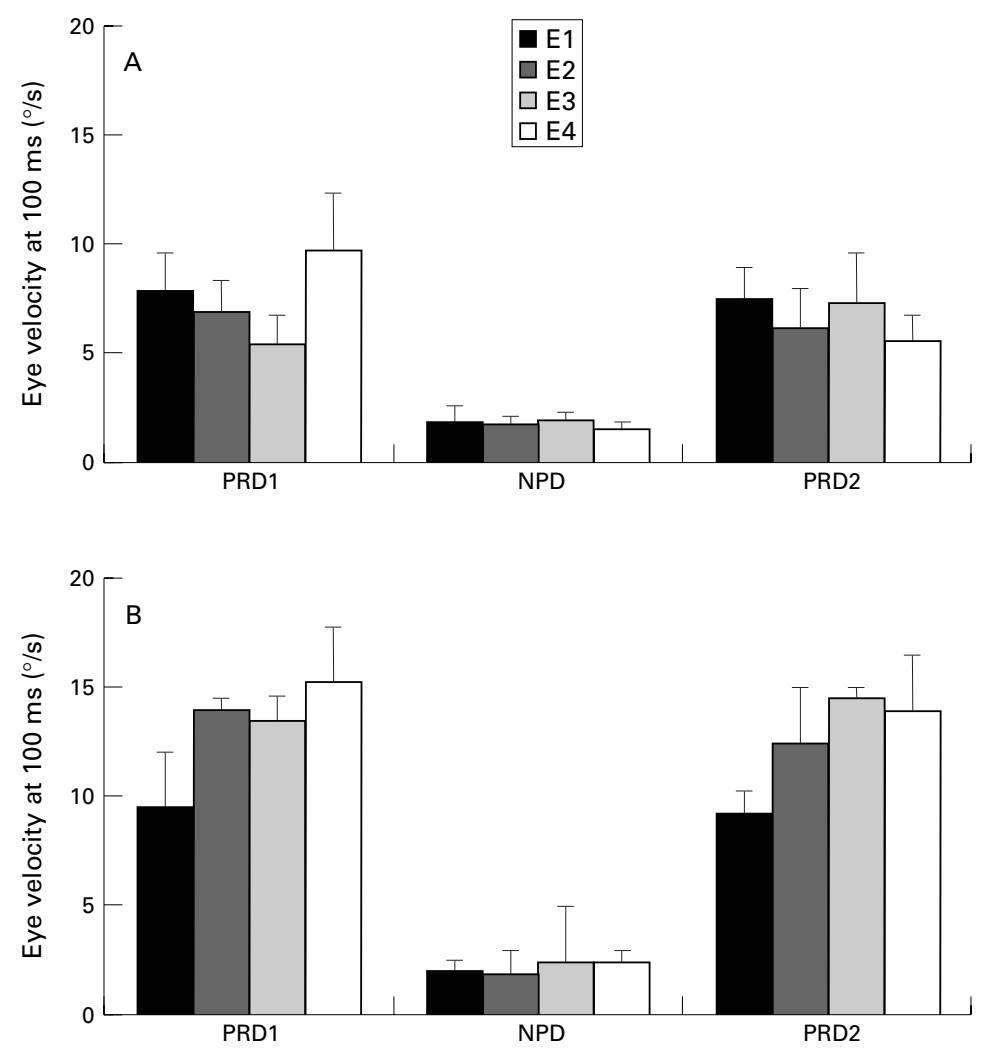

Figure 5 Anticipatory eye velocity (V100) measured $100 \mathrm{~ms}$ after target onset in the four epochs (E1-E4) of each stimulus condition (PRD1, NPD, PRD2) for controls and patients with Parkinson's disease. Each value is the mean (SEM) across nine responses in two directions for seven subjects.

Table 3 Results of separate one way ANOVAs for the specified variables (peak velocity, RMS displacement, V100, V200, and onset latency) in each group (patients with Parkinson's disease (PD) and controls) and each condition (PRD1, NPD, and PRD2). The tested factor was Epoch $(E 1, E 2, E 3, E 4)$. Significance indicated by ${ }^{*}(p<0.05)$.

\begin{tabular}{llllll}
\hline $\begin{array}{l}\text { One way ANOVA } \\
\text { Factor:epoch }\end{array}$ & Peak velocity & RMS Displ & V100 & V200 & $\begin{array}{l}\text { Onset } \\
\text { latency }\end{array}$ \\
\hline $\begin{array}{l}\text { Controls: } \\
\text { (df=1,6) }\end{array}$ & $F=3.37$ & $F=0.573$ & $F=1.736$ & $F=3.005$ & $F=0.013$ \\
PRD1 & $\mathrm{p}=0.116$ & $\mathrm{p}=0.478$ & $\mathrm{p}=0.236$ & $\mathrm{p}=0.134$ & $\mathrm{p}=0.915$ \\
NPD & $F=0.871$ & $F=5.043$ & $F=5.712$ & $F=0.982$ & $F=1.723$ \\
PRD2 & $\mathrm{p}=0.387$ & $\mathrm{p}=0.066$ & $\mathrm{p}=0.054$ & $\mathrm{p}=0.36$ & $\mathrm{p}=0.237$ \\
Patients with Parkinson's & & & & & \\
$\quad$ disease: & $F=0.254$ & $F=0.159$ & $F=6.448$ & $F=2.016$ & $F=0.059$ \\
(df=1,6) & $\mathrm{p}=0.633$ & $\mathrm{p}=0.704$ & $\mathrm{p}=0.044 \star$ & $\mathrm{p}=0.205$ & $\mathrm{p}=0.816$ \\
PRD1 & $F=0.26$ & $F=1.263$ & $F=0.889$ & $F=0.026$ & $F=4.166$ \\
NPD & $\mathrm{p}=0.629$ & $\mathrm{p}=0.304$ & $\mathrm{p}=0.382$ & $\mathrm{p}=0.877$ & $\mathrm{p}=0.087$ \\
PRD2 & $F=6.26$ & $F=3.101$ & $F=0.305$ & $F=0.045$ & $F=1.718$ \\
& $\mathrm{p}=0.046^{\star}$ & $\mathrm{p}=0.129$ & $\mathrm{p}=0.601$ & $\mathrm{p}=0.84$ & $\mathrm{p}=0.238$ \\
& $F=10.435$ & $F=6.73$ & $F=0.355$ & $F=3.392$ & $F=6.234$ \\
& $\mathrm{p}=0.018^{\star}$ & $\mathrm{p}=0.041^{\star}$ & $\mathrm{p}=0.573$ & $\mathrm{p}=0.115$ & $\mathrm{p}=0.047$ \\
\hline
\end{tabular}

${ }^{\star} \mathrm{p}<0.05$.

Analysis of the latency of onset of eye movement showed no significant difference between subject groups (table 2). There were however, differences between conditions. As expected, there was a significant difference in latency between the PRD1 condition, in which movements started before target onset, and the NPD conditions, in which there was a delay before onset of eye movement. But there was also a significant decrease in latency between the PRD1 and PRD2 conditions. Mean latencies (SD) for the controls were -370.4 (182) $\mathrm{ms}$ for PRD1, 108 (36) ms for NPD, and -244 (198) $\mathrm{ms}$ for the PRD2 condition. Corresponding latencies for the patients with Parkinson's disease were -258 (222) ms for PRD1, 134 (51) ms for NPD, and -190 (205) ms for the PRD2 condition.

CHANGES IN RESPONSE ACROSS EPOCHS

To assess trends in the data across epochs 1-4, a series of one way ANOVAs was carried out to determine (using polynomial contrasts) whether there was a linear trend across epochs for each paradigm in each subject group. Table 3 gives details of the results.

For the controls, the results for peak velocity and RMS displacement were similar; there was no linear trend in any of the paradigms (table 3), showing that there was no change in performance over time. This was exemplified by the mean peak velocities (fig 4), which, for $\mathrm{PRD} 1$, ranged from $35.8^{\circ} \mathrm{s}$ in E1 to $40.3 \%$ in E4, and for PRD2 ranged from $39.4 \%$ for E1 to $37.7^{\circ} \mathrm{s}$ in $\mathrm{E} 4$. Likewise, RMS displacement for PRD1 ranged from $8.03^{\circ}$ in $\mathrm{E} 1$ to $8.91^{\circ}$ in $\mathrm{E} 4$ and for PRD2 it ranged from $7.79^{\circ}$ in $\mathrm{E} 1$ to $7.40^{\circ}$ in $\mathrm{E} 4$.

For the Parkinson's disease group, there was also no linear trend for the first predictive paradigm (PRD1) for peak velocity or RMS displacement (table 3). Mean peak velocity ranged from $28.9^{\circ}$ s in $\mathrm{E} 1$ to $27.1 \%$ in $\mathrm{E} 4$ and RMS displacement from $6.52^{\circ}$ in $\mathrm{E} 1$ to $5.52^{\circ}$ in $\mathrm{E} 4$. For NPD, peak velocity did show a significant linear trend, decreasing with time, but RMS displacement did not. But, perhaps the most important result was that, for PRD2, both peak velocity and RMS displacement showed a significant linear trend (table 3 ), with performance deteriorating over time. Thus, for PRD2 mean peak velocity in the patients with Parkinson's disease declined from $31.0 \%$ in E1 to $16.9 \%$ in E4 and RMS displacement went from $6.65^{\circ}$ in $\mathrm{E} 1$ to $3.57^{\circ}$ in $\mathrm{E} 4$.

It is of interest that RMS displacement broke down in a similar way to the peak velocity of smooth pursuit in the PRD2 condition of the patients (fig 1 and 2). It implies that patients did not try to adjust for the deficit in smooth movement by making more saccadic eye movements to maintain the overall amplitude of movement as is commonly seen, for example, in cerebellar patients with reduced smooth pursuit.

Analysis of the latency of onset of eye movement showed no significant change across epochs in the control subjects for any stimulus conditions (table 3). In the patients with Parkinson's disease, however, there was a significant decline in latency across epochs for the PRD2 condition; mean latency decreased from -334 (256) $\mathrm{ms}$ in $\mathrm{E} 1$ to -70 (196) $\mathrm{ms}$ in E4. Thus, as peak eye velocity declined throughout PRD2, the responses became less anticipatory.

The analysis of anticipatory eye velocity (V100) showed a rather different trend to that of peak velocity and latency (fig 5). In the controls there was evidence of a significant linear trend in the PRD2 condition (table 3). V100 increased particularly over the first three epochs (fig 5) A similar trend was present in PRD1, although this was not significant. In the patients with Parkinson's disease there was no linear trend in either PRD1 or PRD2 (table 3). 
Direct comparison of patients and controls within each epoch by independent sample $t$ tests showed that V100 was not significantly different between groups in the first epoch (means across PRD1 and PRD2 of 9.25 $(3.2) \%$ and $7.60(3.9) \%$ in patients with Parkinson's disease). However, in the PRD1 condition, V100 in controls was significantly greater than in patients with Parkinson's disease in $\mathrm{E} 2(\mathrm{df}=12 ; \mathrm{p}=0.042)$ and $\mathrm{E} 3(\mathrm{df}=12$; $\mathrm{p}=0.011$ ) and, in the PRD2 condition, in $\mathrm{E} 4$ $(p=0.018 ; d f=12)$. These effects resulted from subtle changes in the response that are evident in fig 1 . For the controls the velocity profile in the first epoch was discontinuous at $100 \mathrm{~ms}$ but then became smoother in the later epochs, leading to a higher value of V100. In the patients with Parkinson's disease this change did not happen and the discontinuity and lower V100 levels remained throughout all epochs. These subtle changes in anticipatory eye velocity (V100) across epochs were no longer present once visual feedback came into effect, as analysis of eye velocity $200 \mathrm{~ms}$ after target onset (V200) showed no significant difference across epochs (table 3 ).

\section{Discussion}

Our results confirm previous findings of reduced pursuit velocity gain in advanced Parkinson's disease. It is as yet not certain why disorders of the basal ganglia are associated with reduced smooth pursuit gain as the known ocular pursuit pathways do not traverse the basal ganglia. $^{3}$

Our study showed that anticipatory ocular pursuit is preserved in the presence of severe bradykinesia. Inferences of prediction in the pursuit eye movements of Parkinson's disease have been based mostly on changes in phase error. In a study of predictive ability in patients with Parkinson's disease, Flowers ${ }^{23}$ and Flowers and Downing ${ }^{24}$ suggested that although these patients showed evidence of impaired predictive control in a manual task, their predictor mechanism for the control of smooth pursuit eye movements was intact. In another study, Bronstein and Kennard ${ }^{10}$ found no impairment of prediction in the smooth pursuit system of Parkinson's disease. Waterston et al ${ }^{25}$ also found an intact predictive system for smooth pursuit in Parkinson's disease. White et $a l^{11}$ used a predictable ramp stimulus to show the existence of anticipatory pursuit in Parkinson's disease. Their patients achieved anticipatory eye velocities of up to $10 \%$ s. Our study shows that given long extinction intervals (1.248 $\mathrm{s}$ in this study) between periodic ramp target motions, patients with Parkinson's disease can initiate anticipatory pursuit as early as control subjects; and the magnitude of this anticipatory movement forms a similar proportion of peak eye velocity as in controls.

The changes in anticipatory eye velocity (V100) across epochs did not show a similar decline to peak velocity. However, the results in controls showed that prolonged repetition of the ramp stimulus can lead to a progressive increase in V100. This build up of the response should not be confused with the build up that occurs within the first three to four presentations that we have reported before. ${ }^{19}$ In fact, we are not aware that this more prolonged build up has been described before. It seems to represent a gradual process of smoothing out the response around the time when visual feedback would be expected (fig 1 (B)). This trend was not present in the patients. However, before suggesting that this represents a failure of the patients to accomplish this smoothing process, it should also be noted that because of declining peak velocity in the patients, the ratio of V100 to peak velocity actually increases in a similar way in both controls (25\% in E1 to $37 \%$ in E4) and patients with Parkinson's disease (25\% to 34\%).

This study shows that in addition to the nonspecific reduction in smooth pursuit gain seen in Parkinson's disease, there is a characteristic progressive bradykinesia and hypokinesia. Our control subjects maintained a stable peak velocity and displacement in each of the four epochs in the three pursuit tasks, PRD1, NPD, and PRD2. Patients with Parkinson's disease maintained a stable pursuit peak velocity in only the first predictive task (PRD1). However, in the subsequent pursuit tasks (NPD and PRD2) there was a progressive deterioration in velocity and amplitude across the epochs. Progressive bradykinesia and hypokinesia are well known in the skeletal movements of Parkinson's disease. ${ }^{2}$ It is well illustrated in the simple clinical bedside test of repetitive opposition of the thumb and index finger and in micrographia.

Patients with Parkinson's disease are known to have greater difficulty with motor tasks when visual cues ${ }^{26}$ or auditory cues ${ }^{27}$ are lacking. Martin et $a l^{26}$ showed that without external cues, movements in Parkinson's disease deteriorate down the sequence in an additive fashion. When faced with predictable motor sequences, the basal ganglia are said to provide a non-specific internal cue which is necessary for switching between one movement and the next in the sequence and also for development of preparatory activity for each submovement in the sequence. ${ }^{27}$ Our pursuit tasks were either predictable or non-predictable with no external cues in between presentations. Predictability of the task definitely helped the patients to enhance the oculomotor response above the level in the non-predictable conditions but did not help overcome the progressive bradykinesia. The reason for progressive bradykinesia and hypokinesia is not fully understood. One of the explanations is that patients with Parkinson's disease are prone to easy fatigability. ${ }^{28} 29$ This view is supported by our finding that such patients maintained a steady peak velocity in PRD1, but that this progressively deteriorated in the subsequent pursuit tasks, both nonpredictive (NPD) and predictive (PRD2).

Dr GU Lekwuwa was supported by the British Postgraduate Medical Federation and the Medical Research Council (UK).

1 Oertel WH, Quinn NP. Parkinsonism. In: Brandt T, Caplan LR, Dichgans J, et al, eds. Neurological disorders: course and treatment. San Diego, CA: Academic Press, 1996:715-72.

2 Marsden CD. Which motor disorder in Parkinson's disease indicates the true motor function of the basal ganglia? In: Evered D, O'Connor M, eds. Functions of the basal ganglia. London: Pitman, 1984:225-41. 
3 Kennard C, Lueck CJ. Oculomotor abnormalities in diseases of the basal ganglia. Rev Neurol (Paris) $1989 ; 145$ diseases of

4 Melvill Jones G, De Jong BM. Dynamic characteristics of saccadic eye movements in Parkinson's disease. Exp Neurol $1971 ; 31: 17-31$

5 Stell R, Bronstein AM. Eye movement abnormalities in extrapyramidal diseases. In: Marsden CD, Fahn S, eds. Movement disorders 3. Oxford: Butterworth-Heinemann, 1994:88-113.

6 Ventre J, Zee DS, Papageorgiou H, et al. Abnormalities of predictive saccades in hemi-Parkinson's disease. Brain 1992;115: 1147-65.

7 Waterston JA, Barnes GR, Grealy MA, et al. Abnormalities of smooth eye and head movement control in Parkinson's disease. Ann Neurol 1996;39:749-60.

8 Rottach KG, Riley DE, DiScenna AO, et al. Dynamic properties of horizontal and vertical eye movements in
parkinsonian syndromes. Ann Neurol 1996;39:368-77.

9 Lueck CJ, Tanyeri S, Crawford TJ, et al. Antisaccades and remembered saccades in Parkinson's disease. 7 Neurol Neuremembered saccades in Parkins
rosurg Psychiatry 1990;53:284-8.

rosurg Psychiatry 1990;53:284-8.
10 Bronstein AM, Kennard C. Predictive ocular motor control in Parkinson's disease. Brain 1985;108:925-40.

11 White OB, Saint-Cyr JA, Tomlinson RD, et al. Ocular motor deficits in Parkinson's disease II. Control of the saccadic and smooth pursuit systems. Brain 1983;106:571-87.

12 Sharpe JA, Fletcher WA, Lang AE, et al. Smooth pursuit during dose-related on-off fluctuations in Parkinson's disease. Neurology 1987;37:1389-92

13 Gibson JM, Pimlott R, Kennard C. Ocular motor and manual tracking in Parkinson's disease and the effect of treatment. $\mathcal{7}$ Neurol Neurosurg Psychiatry 1987;50:853-60.

14 Rascol O, Clanet M, Montastruc JL, et al. Abnormal ocular movements in Parkinson's disease: evidence for involvement of dopaminergic mechanisms. Brain 1989;112:1193214.

15 Flowers KA. Some frequency response characteristics of parkinsonism on pursuit tracking. Brain 1978;101:19-34.

16 Vidailhet M, Rivaud S, GouiderKhouja N, et al. Eye movements in parkinsonian syndromes. Ann Neurol 1994;35 $420-6$.
17 Alexander GE, DeLong MR, Strick PL. Parallel organization of functionally segregated circuits linking basal ganglia and cortex. Annu Rev Neurosci 1986;9:357-81.

18 Hikosaka O. Role of basal gangia in saccades. Rev Neurol (Paris) 1989;145:580-6.

19 Barnes GR, Asselman PT. The mechanism of prediction in human smooth pursuit eye movements. F Physiol (Lond) 1991;439:439-61.

20 Lekwuwa GU, Barnes GR. Cerebral control of eye movements: timing of anticipatory eye movements, predictive pursuit, and phase errors in focal cerebral lesions. Brain 1996;119:491-505.

21 CAPIT Committee: Langston JW, Widner H, Goetz CE, et al. Core assessment program for intracerebral transplantations (CAPIT). Mov Disord 1992;7:2-13.

22 Barnes GR. A procedure for the analysis of nystagmus and other eye movements. Aviat Space Environ Med 1982;53: 676-82.

23 Flowers KA. Lack of prediction in the motor behaviour of parkinsonism. Brain 1978;101:35-52.

24 Flowers KA, Downing AC. Predictive control of eye movements in Parkinson disease. Ann Neurol 1978;4:63-6.

25 Waterston JA, Barnes GR, Grealy MA. Predictive velocity estimation in the pursuit reflex of patients with cerebellar ataxia and Parkinson's disease. In: d'Ydawalle G, van Rensbergen J, eds. Visual and oculomotor functions. Advances in eye movement research. Amsterdam: North-Holland, 1994:187-97.

26 Martin KE, Phillips JG, Iansek R, et al. Inaccuracy and instability of sequential movements in Parkinson's disease. Exp Brain Res 1994;102:131-40.

27 Georgiou N, Iansek R, Bradshaw JL, et al. An evaluation of the role of internal cues in the pathogenesis of parkinsonian hypokinesia. Brain 1993;116:1575-87.

28 Friedman J, Friedman H. Fatigue in Parkinson's disease. Neurology 1993;43:2016-18.

29 van Hilten JJ, Hoogland G, van der Velde EA, et al. Diurnal effects of motor activity and fatigue in Parkinson's disease. f Neurol Neurosurg Psychiatry 1993;56:874-7. 